\title{
Buen o buena docente de universidad: Perspectiva del personal directivo de carrera y de los mismos grupos docentes ${ }^{1}$
}

\author{
Being a Good University Teacher: Perspective of Program Directors and Teachers Themselves² \\ Boa professora ou bom professor universitário: Perspectiva das pessoas coordenadoras de \\ carreiras e da equipe docente ${ }^{3}$
}

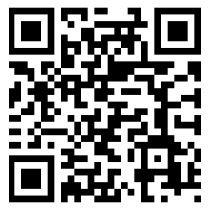

\author{
Rosa Orellana-Fernández ${ }^{4}$ \\ Universidad Autónoma de Chile \\ Talca, Chile \\ rosa.orellana@uautonoma.cl
}

Eugenio Merellano-Navarro

Universidad Autónoma de Chile

Talca, Chile

emerellanon@uautonoma.cl

Alejandro Almonacid-Fierro ${ }^{6}$

Universidad Autónoma de Chile

Talca, Chile

aalmonacidf@uautonoma.cl

Recibido • Received • Recebido: 27 / 01 / 2017

Corregido • Revised • Revisado: 02 / 02 / 2018

Aceptado • Accepted • Aprovado: 19/ 02 / 2018

\footnotetext{
${ }^{1}$ Este artículo se deriva de una investigación titulada Caracterización de un buen docente universitario según la percepción de estudiantes, docentes y directores de carrera de la Facultad de Educación de la Universidad Autónoma de Chile-Talca. Trabajo financiado por la Dirección de Investigación y Postgrado de la Universidad Autónoma de Chile, código DPI26-2015.

${ }^{2}$ This article is derived from a research project named "Characterization of a good university teacher according to the perception of student, teacher and directors of the Faculty of Education of the Universidad Autónoma Chile - Talca". This project is funded by the internal Fund of Research at the Autonomous University of Chile, code DPI26-2015.

${ }^{3}$ Este artigo é derivado de uma pesquisa intitulada: Caracterização de um bom professor universitário, de acordo com a percepção dos estudantes, professores e coordenadores de carreira na Faculdade de Educação da Universidade de Chile-Talca. Trabalho financiado pelos Estudos de Pesquisa e Pós-Graduação da Universidade Autônoma de Chile, código DPI26-2015.

${ }^{4}$ Doctora en Ciencias de la Educación de la Universidad Paris Ouest-Nanterre La Defense, Francia. Docente de la Facultad de Educación de la Universidad Autónoma de Chile, sede Talca. Experiencia como investigadora en el área de los proyectos educativos.

${ }^{5}$ Doctor de la Universidad de Extremadura, España. Docente asistente de la Universidad Autónoma de Chile, sede Talca. Experiencia como investigador en el área de la didáctica de la educación física.

${ }^{6}$ Doctor de la Universidad de Extremadura, España. Docente asociado de la Universidad Autónoma de Chile, sede Talca. Experiencia como investigador en el área de formación del profesorado.
} 
doi: http://dx.doi.org/10.15359/ree.22-2.6

URL: http://www.una.ac.cr/educare

CORREO: educare@una.cr

Resumen: Este artículo de investigación se sitúa en la prolongación del estudio de caso sobre la percepción de la calidad docente desde la mirada de estudiantes de la Facultad de Educación de la Universidad Autónoma de Chile, sede Talca. Se pretende aportar una comprensión del fenómeno desde la óptica de los diversos actores involucrados. Esta segunda parte de la investigación apunta a conocer las características de un buen o una buena docente de universidad, según la opinión de los mismos grupos docentes y directivos pertenecientes a esta Facultad. La metodología adoptada es cualitativa y de estudio de caso. Los datos recogidos, por medio de la técnica de grupos focales de docentes y personal directivo de carrera, se sometieron a un análisis de contenido, asistido por el programa Nvivo 11. Se procedió a una codificación de clasificación con categorías previas y emergentes. De este proceso se desprenden finalmente cuatro dimensiones de análisis: características humanas, ideológicas, técnico-pedagógicas y profesionalesinstitucionales. Se concluye que la buena persona docente posee, principalmente, características éticas, morales y afectivas que estructura las interacciones con el estudiantado. Se reconocen como principales atributos de la docencia de calidad la permanente curiosidad, búsqueda del saber y diálogo entre las distintas formas de conocimiento. Características técnico-pedagógicas que asoman en esta investigación como la adaptación metodológica de la enseñanza y la evaluación emergen como aspectos secundarios a la hora de calificarle. Se pone en relieve que, para docentes y equipos directivos, lo humano y lo ideológico se sitúan por sobre lo técnico-pedagógico y lo institucional.

Palabras claves: Docente; enseñanza superior; formación de docentes.

Abstract: This research article represents a prolongation of the case study on the perception of a good university teacher from the perspective of the Education Faculty students at Universidad Autónoma de Chile (Autonomous University of Chile), Talca. It intends to contribute with a different understanding of the phenomenon from the perspective of the actors involved. This second part of the research aims to disclose the characteristics of a good University Teacher according to the opinion of the teachers themselves and administrators belonging to that Faculty. The methodology in approach adopted consisted of a qualitative one as well as of a case study. Through a process of categorization and codification, four characteristic dimensions of analysis were obtained: human, ideological, technicalpedagogical and professional-institutional. It is concluded that a good University Teacher has mainly ethical, moral and affective features that structure the interactions with the students' community. Concerning the main attributes of quality teaching, the permanent curiosity, search for knowledge, and dialogue between the different forms of it are recognized. Technical-pedagogical characteristics that appear in this research as the methodological adaptation of teaching and evaluation emerge as secondary aspects when it comes to qualifying a good University Teacher.

Keywords: Higher education; teachers; teacher education.

Resumo: Esta pesquisa está enfocada na continuidade do estudo de caso sobre a percepção da qualidade dos y das docentes a partir da perspectiva dos estudantes da Faculdade de Educação, na Universidade Autónoma de Chile, região de Talca. O propósito é ajudar na compreensão do fenômeno, desde o ponto de vista das diversas partes interessadas. Esta segunda parte da pesquisa tem como objetivo conhecer as características de um bom ou uma boa professora universitária, de acordo com a opinião dos mesmos professores e coordenadores desta Faculdade. A metodologia utilizada é qualitativa e estudo de caso. Os dados coletados por meio da técnica de grupos focais de professores e coordenadores de carreira, foram submetidos a análises de conteúdo, ajudado pelo programa Nvivo 11. Procedeu-se a uma codificação de classificação de categorias previas e emergentes. a partir de este processo, surgiram quatro dimensões de análise: características humanas, ideológicas, 


\begin{abstract}
técnico-pedagógicas e profissionais-institucionais. Como conclusão, para considerar ser um bom o boa professora, estes devem possuir características éticas, morais e emocionais que estruturam as interações com os estudantes. As qualidades e atribuições que devem existir nas pessoas docentes são uma permanente curiosidade como também a busca de conhecimento e diálogo entre diferentes formas de conhecimento. As características técnicas-pedagógica que aparecem nessa pesquisa, como a adaptação metodológica de ensino e avaliação são consideradas aspectos secundários no momento de ser qualificado. Como ponto de destaque tanto para os professores como equipe coordenadora, a parte humana e ideológica são mais importantes que o aspecto técnico-pedagógico e institucional.
\end{abstract}

Palavras-chave: Docência; ensino superior; formação de professores.

\title{
Introducción
}

La docencia universitaria está llamada a renovarse adoptando paradigmas que le permitan aproximarse a la complejidad de nuestro siglo en los distintos contextos a nivel mundial. Los factores de este cambio están vinculados principalmente al entorno político, económico, social, cultural y factores internos de cada institución (Gibezzi, 2010). La docencia en educación superior debe hoy construirse a sí misma en su andar y a partir de sus propias incertidumbres y exigencias. En un intento pedagógico hermenéutico el profesorado universitario se vuelve también un actor en la producción de nuevos conocimientos sobre la docencia (García y Martín, 2013).

En Chile, como en el resto del mundo, se vive un proceso de masificación (Palma, 2013) que conduce a generar cambios en diversos ámbitos. Las exigencias académicas en la formación profesional en el siglo veintiuno han transformado las orientaciones curriculares convencionales por un modelo basado en el desarrollo de competencias diversas en el nuevo perfil profesional (González, Herrera y Zurita, 2008). A esto podría sumarse que no solo el contexto y la universidad y su currículo cambian, sino que también sus estudiantes. Estos son jóvenes con una expresión más libre, con acceso a una mayor información y con un cómodo manejo de las tecnologías de la información y comunicación (TIC), la llamada generación "Y" (Demougeot-Lebel, 2014). Estos nuevos escenarios, a su vez, interrogan las formas de enseñar y de aprender en la universidad.

Para responder a estos fenómenos los modelos pedagógicos se multiplican, yendo desde una exclusiva centralidad en el contenido hacía una docencia focalizada en el estudiantado. En el caso de la formación de docentes del futuro, esta cuestión no es baladí. De la Cruz et al. (2006, citado por De-Juanas, Esquerra y Martín, 2016) plantean que las concepciones acerca de la enseñanza de docentes de universidad van "'impregnando'a los estudiantes, que serán profesores de primaria o secundaria. De tal manera, los docentes universitarios que forman profesores también enseñan a enseñar, enseñando" (p. 394). En este sentido, el personal docente universitario exige la necesidad de conocer, comprender las individualidades del estudiantado, principalmente en los ritmos y sistemas de aprendizaje más efectivos para el contexto en el que se trabaja. Francis (2006) expone que el profesorado universitario debe tener la capacidad de influir en la formación del estudiantado mediante los procesos de enseñanza-aprendizaje, con el propósito de trascender. Por su parte, Tardif (2004) define al profesorado ideal como aquel que conoce su área 
doi: http://dx.doi.org/10.15359/ree.22-2.6

URL: http://www.una.ac.cr/educare

CORREO: educare@una.cr

disciplinar, y que además de ello, posee ciertos conocimientos relativos a aspectos transversales de la pedagogía, desarrollando un saber práctico vinculado al perfil de egreso, basado en la experiencia personal y del estudiantado. Desde esta perspectiva, el accionar docente no se debe limitar a trasferir conocimientos, sino a crear y otorgar posibilidades de su propia construcción. A través de esto, se superará la relación clásica de sujeto-objeto (profesorado-alumnado): es el mismo personal docente que, al "formar", se re-forma, se replantea desde el estudiantado.

Reconociendo la relevancia que tiene el profesorado y lo trascendente que debe ser el proceso formativo del futuro profesorado, es importante conocer la percepción del buen o buena docente desde la mirada de todos los actores que participan en el proceso. A pesar de que existen muchos estudios referidos al tema (Casero, 2010; Fernández y González, 2012; Luna-Serrano, ValleEspinosa y Osuna-Lever, 2010; Martínez, García y Quintana, 2006; Merellano-Navarro, AlmonacidFierro, Moreno-Dona y Castro-Jaque, 2016), aún no hay un conceso sobre cómo debe ser un buen profesorado y, más aún, el buen o buena docente que dicta clases en la universidad. Estos desacuerdos generan un debate permanente referido al conjunto de cuestiones metodológicas que orientan y guían el accionar del profesorado. Estas investigaciones analizadas centran su atención en pesquisar cuales son las características que debe poseer la persona docente universitaria desde la opinión del estudiantado. Existe poca información sobre lo que cada docente considera relevante para realizar una docencia de calidad. A diferencia de los trabajos presentados anteriormente, Rocha (2012) describe las diferencias entre las perspectivas del estudiantado y el equipo de docentes de la docencia universitaria. Si bien este trabajo no aborda al buen o buena docente desde la totalidad de los actores que influyen en el proceso formativo, es el único estudio encontrado que incorpore a más de un actor del proceso educativo, en este caso, docentes y estudiantes.

Este trabajo es parte de un estudio mayor que tiene por objetivo caracterizar al "buen docente universitario" desde la perspectiva de diversos actores. La primera parte de este estudio advierte que los rasgos interpersonales y el dominio del contenido son altamente valorados por el estudiantado. Esto implica la capacidad del personal docente de conjugar lo pedagógico, lo humano y lo ideológico en su propósito formativo (Merellano-Navarro, et al., 2016). En esta segunda parte de la investigación, la muestra se extiende hacia el cuerpo académico. El objetivo es analizar las concepciones del mismo personal docente, directivos y directivas de carrera de la Facultad de Educación de la Universidad Autónoma de Chile, sede Talca, con respecto a las características que debe poseer quien se desempeñe como buen o buena docente de universidad. El análisis se centra en las concepciones declaradas y no en la puesta en práctica efectiva de esos principios declarados.

\section{Metodología}

La metodología es cualitativa y se optó por el estudio de caso (Simons, 2011) de docentes pertenecientes a la Facultad de Educación de la Universidad Autónoma de Chile, sede Talca, Chile. Esta Facultad imparte "las carreras de Pedagogía en las siguientes especialidades: Artes 
Visuales, Matemática, Inglés, Lengua Castellana y Comunicación, Educación Física, Educación General Básica, Historia Geografía y Ciencias Sociales y Educación Parvularia" (MerellanoNavarro, et al., 2016). Para la recolección de información, se optó por la técnica de grupos focales de personal docente y directivo de carreras impartidas en Facultad (Tabla 1). Estos grupos apuntaban a generar un espacio de conversación con el fin de conocer el sentir, pensar y el vivir de sus participantes (Hamui-Sutton y Varela-Ruiz, 2013).

Tabla 1: Total de participantes grupos focales

\begin{tabular}{lcc}
\hline \multicolumn{1}{c}{ Carreras Facultad de Educación } & $\begin{array}{c}\text { Grupo focal Personal } \\
\text { docente }\end{array}$ & $\begin{array}{c}\text { Grupo focal } \\
\text { Personal directivo }\end{array}$ \\
\hline Pedagogía en Educación General Básica & 1 & 1 \\
Pedagogía en Educación Física & 1 & 1 \\
Pedagogía en Educación Parvularia & 1 & 1 \\
Pedagogía en Inglés & 1 & 1 \\
Pedagogía en Historia Geografía y Ciencias Sociales & 1 & 1 \\
Pedagogía en Lengua Castellana y Comunicación & 1 & 1 \\
Pedagogía en Matemática & 1 & 1 \\
Pedagogía en Artes Visuales & 1 & 1 \\
\hline TOTAL & 8 & 8 \\
\hline
\end{tabular}

Nota: Elaboración propia.

La selección de los sujetos que participaron en la investigación se realizó a través del cumplimiento de los siguientes criterios: a) personal docente adscrito a una de las especialidades que imparte la Facultad de Educación; b) poseer carga académica durante el semestre que se realizó el estudio; y c) interés en participar en el estudio. En el grupo focal de personal directivo se agregó el criterio de: poseer cargo directivo en alguna de las carreras que pertenecen en la Facultad de Educación. Las personas participantes en ambos grupos focales pertenecen a todas las carreras de la Facultad de Educación. Ambos grupos focales se realizaron durante el periodo el primer trimestre del 2016, con su previa autorización a través de la firma de un consentimiento.

\section{Procedimiento de análisis}

Los datos recogidos fueron tratados según el siguiente procedimiento. Los dos grupos focales, uno realizado con personal directivo y otro con docentes de cada una de las carreras, 
doi: http://dx.doi.org/10.15359/ree.22-2.6

URL: http://www.una.ac.cr/educare

CORREO: educare@una.cr

fueron transcritos integralmente. Ambos recursos fueron sometidos a un análisis de contenido (Voynnet, 2012) mediante codificación de unidades de significado de los textos (Blais y Martineau, 2006). Estos datos fueron analizados mediante una codificación doble (dos sujetos investigadores). Se inició el proceso con una codificación de primer nivel de clasificación. Para dicho efecto los elementos del discurso fueron organizados en códigos temáticos por la primera persona investigadora. En un primer momento los códigos fueron clasificados en tres categorías previas establecidas en un estudio precedente por Ventura, e Neves, Loureiro, Frederico-Ferreira y Cardoso (2011): características técnicas/pedagógicas; características ideológicas; características humanas.

A partir de esta primera codificación emergen tres nuevas categorías: trayectoria profesional, especialización e institución. Una segunda persona investigadora realizó este mismo procedimiento de codificación de clasificación de los datos de donde emerge como cuarta categoría: características profesionales-institucionales. Más tarde los códigos fueron reagrupados en subcategorías (ver Tabla 2) para cada categoría previa (Saldaña, 2009).

Estas dos etapas de investigación fueron asistidas por el programa de análisis de datos cualitativos Nvivo 11. Esta herramienta permite organizar los datos en recursos (fuentes de información), en este caso, las transcripciones de los grupos focales. También permite evaluar y reajustar la codificación de manera permanente, así como también recontextualizar las referencias codificadas (relatos a los que se les atribuyó un código). Además, el programa permite extraer del análisis de los datos diversas síntesis visuales: matrices, gráficos, diagramas, mapas conceptuales que facilitan la comunicación y comprensión de la información.

\section{Análisis de los datos y resultados de la investigación}

A continuación, se presenta la caracterización del buen personal docente universitario desde la perspectiva de docentes y equipos directivos de la carrera de la Facultad de Educación. Esta se obtiene luego del análisis de los datos mediante el proceso de codificación de datos. Estos permiten caracterizar al personal docente según cuatro dimensiones: humanas, ideológicas, técnico-pedagógicas y profesionales-institucionales.

En un primer momento se presentarán las cuatro dimensiones que constituyen las categorías previas de clasificación, de donde se desprenden subcategorías (mayor nivel de abstracción) y sus códigos descendentes primarios y secundarios (menor nivel de abstracción). Luego de la revisión de cada una de las dimensiones, se profundizará en los aspectos en los que ponen mayor énfasis los grupos docentes y directivos, con el fin de establecer elementos de comparación. La cuarta parte de este artículo profundizará desde una lógica interpretativa y presentará en detalle los elementos más relevantes obtenidos de este análisis. Cabe señalar que se presenta la totalidad de referencias codificadas en la investigación (ver Tabla 2).

6 Rosa Orellana-Fernández, Eugenio Merellano-Navarro y Alejandro Almonacid-Fierro

Los artículos de la Revista Electrónica Educare del Centro de Investigación y Docencia en Educación de la Universidad Nacional, Costa Rica, se comparten bajo términos de la Licencia Creative Commons: Reconocimiento, No Comercial, Sin Obra Derivada 3.0 Costa Rica. Las autorizaciones adicionales a las aquí delimitadas se pueden obtener en el correo: educare@una.cr 
doi: http://dx.doi.org/10.15359/ree.22-2.6

URL: http://www.una.ac.cr/educare

CORREO: educare@una.cr

Tabla 2: Matriz de sistematización de categorías

\begin{tabular}{|c|c|c|c|c|c|}
\hline 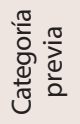 & Subcategoría & Códigos & Subcódigos & Referencia & $\%$ \\
\hline \multirow{13}{*}{ 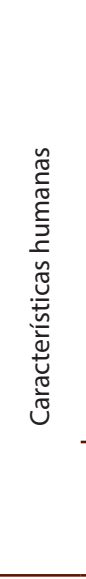 } & \multirow{10}{*}{ Ejemplo de alteridad } & \multirow[t]{3}{*}{ Intrínsecamente altruista } & Comprometido & 6 & 3.6 \\
\hline & & & Empático & 7 & 4.2 \\
\hline & & & Paciente & 4 & 2.4 \\
\hline & & \multirow[t]{6}{*}{ Próximo al estudiantado } & Cercano & 5 & 3.0 \\
\hline & & & Conoce & 3 & 1.8 \\
\hline & & & De buen trato & 1 & 0.6 \\
\hline & & & Humilde & 4 & 2.4 \\
\hline & & & Privilegia la comunicación & 7 & 4.2 \\
\hline & & & Sonriente & 1 & 0.6 \\
\hline & & Se adapta al contexto & & 7 & 4.2 \\
\hline & \multirow{3}{*}{ Produce efecto pigmalión } & Favorece la autoestima & & 2 & 1.2 \\
\hline & & Incita ganas de aprender & & 3 & 1.8 \\
\hline & & Manifiesta altas expectativas & & 3 & 1.8 \\
\hline \multirow{17}{*}{ 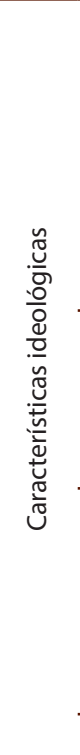 } & \multirow{7}{*}{ Es capaz de trascender } & Cambia una vida & & 1 & 0.6 \\
\hline & & Cautiva & & 1 & 0.6 \\
\hline & & Consecuente & & 1 & 0.6 \\
\hline & & Encarna valores & & 3 & 1.8 \\
\hline & & Muestra rigurosidad & & 4 & 2.4 \\
\hline & & Es modelo & & 5 & 3.0 \\
\hline & & Logra aprendizajes significativos & & 3 & 1.8 \\
\hline & \multirow{4}{*}{ Es epistemológicamente coherente } & Debe producir conocimiento & & 1 & 0.6 \\
\hline & & Manifiesta erudición en su tema & & 7 & 4.2 \\
\hline & & No separa teoría y práctica & & 8 & 4.8 \\
\hline & & Valora la teoría para comprender la práctica & & 4 & 2.4 \\
\hline & \multirow{5}{*}{ Mantiene una relación abierta con el saber } & Trabaja con pasión & & 1 & 0.6 \\
\hline & & Muestra autonomía & & 1 & 0.6 \\
\hline & & Tiene actitudes de curiosidad & & 1 & 0.6 \\
\hline & & Es una persona reflexiva & & 3 & 1.8 \\
\hline & & Transversal & & 2 & 1.2 \\
\hline & Tiene vocación & & & 7 & 4.2 \\
\hline \multirow{7}{*}{ 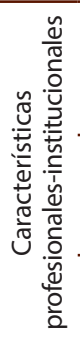 } & \multirow{3}{*}{ Tiene experticia disciplinaria } & Es visible & & 2 & 1.2 \\
\hline & & Se actualiza & & 7 & 4.2 \\
\hline & & Se especializa & & 6 & 3.6 \\
\hline & \multirow[b]{2}{*}{ Tiene trayectoria profesional integral } & Tiene diplomas & & 1 & 0.6 \\
\hline & & $\begin{array}{l}\text { Tiene experiencia profesional en lo que } \\
\text { enseña }\end{array}$ & & 6 & 3.6 \\
\hline & \multirow{2}{*}{ Zonas de tensión } & La evaluación docente debe adaptarse & & 8 & 4.8 \\
\hline & & Trabaja colaborativamente & & 1 & 0.6 \\
\hline
\end{tabular}


doi: http://dx.doi.org/10.15359/ree.22-2.6

URL: http://www.una.ac.cr/educare

CORREO: educare@una.cr

\begin{tabular}{|c|c|c|c|c|c|}
\hline 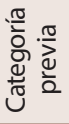 & Subcategoría & Códigos & Subcódigos & Referencia & $\%$ \\
\hline \multirow{13}{*}{ 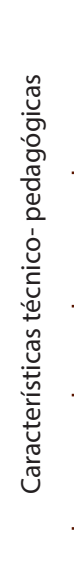 } & \multirow{4}{*}{ Adapta su metodología } & Considera la diversidad de perfiles & & 1 & 0.6 \\
\hline & & Considera los tiempos para aprender & & 1 & 0.6 \\
\hline & & Maneja estrategias diversas & & 3 & 1.8 \\
\hline & & Redirecciona acciones & & 1 & 0.6 \\
\hline & \multirow{3}{*}{ Domina la evaluación } & Diseña instrumentos pertinentes & & 2 & 1.2 \\
\hline & & Evalúa lo que enseña & & 2 & 1.2 \\
\hline & & Retroalimenta los aprendizajes & & 3 & 1.8 \\
\hline & \multirow{2}{*}{ Facilita el aprendizaje } & Genera un clima grato & & 4 & 2.4 \\
\hline & & Metodología participativa & & 1 & 0.6 \\
\hline & \multirow{3}{*}{ Motiva el razonamiento } & Desarrolla pensamiento crítico & & 4 & 2.4 \\
\hline & & Relaciona teoría y práctica & & 2 & 1.2 \\
\hline & & Hace ver lo complejo fácil & & 1 & 0.6 \\
\hline & Tiene objetivos definidos & & & 4 & 2.4 \\
\hline
\end{tabular}

Nota: Elaboración propia.

\section{Caracterización del buen personal docente según grupos docentes y directivos}

Los elementos constitutivos de cada característica son presentados a través de matrices que indican la categoría previa y subcategorías con sus respectivos códigos descendentes. En la columna central se ubica el número de recursos asociados que son las fuentes desde donde se extraen las referencias codificadas. La columna derecha indica el número de referencias codificadas de manera general por categoría y subcategoría, y de manera específica por cada código.

\section{Características humanas}

Esta categoría corresponde a la dimensión ética, moral y afectiva que estructura las interacciones humanas entre docente y estudiante: un saber-ser y un saber vivir junto a la otra persona (Delors, 1997). Se trata, entonces, del marco valórico y actitudinal en el que se desenvuelve el personal docente y el que le permite construir relaciones interpersonales humanamente simétricas con sus estudiantes.

La categoría previa características humanas concentra 91 referencias codificadas (ver Tabla 3). De ella se desprenden dos subcategorías: Ejemplo de alteridad y Produce efecto pigmalión. Es posible observar en la Tabla 3 los códigos y subcódigos que constituyen cada una de estas subcategorías junto con el porcentaje de referencias para cada una de ellas.

De acuerdo con el porcentaje de referencias codificadas, se puede concluir que, en lo que respecta a la caracterización humana del buen personal docente, ejemplo de alteridad reúne la 
mayor cantidad de referencias. Los códigos próximos al estudiantado y el código intrínsecamente altruista aparecen como los elementos más significativos para describir el su altruismo a partir de la perspectiva del profesorado y personal directivo de la Facultad de Educación de la Universidad Autónoma de Chile.

Tabla 3: Características humanas

\begin{tabular}{lcc}
\hline \multicolumn{1}{c}{ Característica } & Recursos & Referencias \\
\hline 1. Características humanas & $\mathbf{2}$ & 91 \\
1.1 Ejemplo de alteridad & 2 & \\
1.1.1 Intrínsecamente altruista & 2 & 17 \\
1.1.1.1 Comprometido/a & 2 & 6 \\
1.1.1.2 Empático/a & 2 & 7 \\
1.1.1.3 Paciente & 1 & 4 \\
1.1.2 Próximo/a al estudiantado & 2 & 21 \\
1.1.2.1 Cercano/a & 2 & 5 \\
1.1.2.2 Conoce & 1 & 3 \\
1.1.2.3 De buen trato & 1 & 1 \\
1.1.2.4 Humilde & 1 & 4 \\
1.1.2.5 Privilegia la comunicación & 2 & 7 \\
1.1.2.6 Sonriente & 1 & 1 \\
1.1.3 Se adapta al contexto & 2 & 7 \\
\hline 1.2 Produce efecto pigmalión & 2 & 3 \\
1.2.1 Favorece la autoestima & 1 & 2 \\
1.2.2 Incita ganas de aprender & 1 & 3 \\
1.2.3 Manifiesta altas expectativas & 2 & 3 \\
\hline & & 3 \\
\hline
\end{tabular}

Nota: Elaboración propia. 
doi: http://dx.doi.org/10.15359/ree.22-2.6

URL: http://www.una.ac.cr/educare

CORREO: educare@una.cr

\section{Características ideológicas}

Tabla 4: Características ideológicas

\begin{tabular}{lcc}
\hline \multicolumn{1}{c}{ Características } & Recursos & Referencias \\
\hline 2. Características ideológicas & $\mathbf{2}$ & $\mathbf{5 3}$ \\
2.1 Es capaz de trascender & $\mathbf{2}$ & \\
2.1.1 Cambia una vida & 1 & 1 \\
2.1.2 Cautiva & 1 & 1 \\
2.1.3 Consecuente & 1 & 1 \\
2.1.4 Encarna valores & 1 & 3 \\
2.1.5 Es riguroso/a & 2 & 4 \\
2.1.6 Es un/a modelo & 1 & 5 \\
2.1.7 Logra aprendizajes significativos & 1 & 3 \\
\hline 2.2. Es epistemológicamente coherente & $\mathbf{2}$ & \\
2.2.1 Debe producir conocimiento & 1 & 1 \\
2.2.2 Erudito/a en su tema & 2 & 7 \\
2.2.3 No separa teoría y práctica & 2 & 8 \\
2.2.4 Valora la teoría para comprender la práctica & 2 & 4 \\
\hline 2.3 Mantiene una relación abierta con el saber & $\mathbf{2}$ & \\
2.3.1 Apasionado/a & 1 & 1 \\
2.3.2 Autónomo/a & 1 & 1 \\
2.3.3 Curioso/a & 1 & 1 \\
2.3.4 Reflexivo/a & 2 & 3 \\
2.3.5 Transversal & 2 & 2 \\
\hline 2.4 Tiene vocación & $\mathbf{1}$ & $\mathbf{7}$ \\
\hline & & \\
\hline
\end{tabular}

Nota: Elaboración propia.

Se clasifican en esta categoría contenidos que tienen que ver con la cultura profesional que rige su actuar dentro y fuera del aula universitaria. Se consideran teorías y creencias que docentes y personal directivo señalan como fundamentos de la acción pedagógica de calidad. Reglas de conducta para educar sobre la que sustenta la relación humana, pero esta vez estableciendo estatus diferentes para quien enseña y quien aprende. 
De esta segunda categoría previa emergen tres subcategorías que tienen que ver con la relación del personal docente universitario con el conocimiento y su práctica y, a la vez, con la manera en cómo es capaz de movilizar al estudiantado en esta dinámica: Es capaz de trascender, Es epistemológicamente coherente y Mantiene una relación abierta con el saber. Las dos primeras reagrupan el mayor porcentaje de referencias codificadas (ver Tabla 4).

\section{Características profesionales e institucionales}

Se encuentran en esta categoría aspectos relacionados con la trayectoria profesional y académica del personal docente, el desarrollo de su carrera profesional y la relación que este mantiene con la institución en la que se inserta laboralmente.

La tercera dimensión Características profesionales-institucionales (verTabla 5) emerge como categoría de clasificación durante la primera etapa de codificación. Esta característica pone en relieve la experticia disciplinaria del personal docente con los códigos: Es visible, Se actualiza y Se especializa. Se destaca en las características profesionales-institucionales la subcategoría Zonas de tensión. Esta subcategoría reúne aspectos críticos señalados por docentes y personal directivo con respecto a los instrumentos institucionales de evaluación del trabajo del profesorado y formas de trabajo condicionadas por la estructura de los equipos académicos.

Tabla 5: Características profesionales e institucionales

\begin{tabular}{lcc}
\hline \multicolumn{1}{c}{ Características } & Recursos & Referencias \\
\hline 3. Características profesionales-institucionales & $\mathbf{2}$ & $\mathbf{3 1}$ \\
3.1 Tiene experticia disciplinaria & $\mathbf{2}$ & \\
3.1.1 Es visible & 1 & 2 \\
3.1.2 Se actualiza & 2 & 7 \\
3.1.3 Se especializa & 2 & 6 \\
\hline 3.2 Tiene trayectoria profesional integral & $\mathbf{2}$ & \\
3.2.1 Tiene diplomas & 1 & 1 \\
3.2.2 Tiene experiencia profesional en lo que enseña & 2 & 6 \\
\hline 3.3 Zonas de tensión & $\mathbf{2}$ & \\
3.3.1 La evaluación docente debe adaptarse & 2 & 8 \\
3.3.2 Trabaja colaborativamente & 1 & 1 \\
\hline
\end{tabular}

Nota: Elaboración propia. 
doi: http://dx.doi.org/10.15359/ree.22-2.6

URL: http://www.una.ac.cr/educare

CORREO: educare@una.cr

\section{Características técnico-pedagógicas}

El conjunto de estas características hace referencia a los saberes, procesos, condiciones y artefactos que el personal docente conoce, desarrolla, transforma y pone a disposición del estudiantado con el fin de facilitar el proceso de enseñanza-aprendizaje y posibilitar aprendizajes significativos. Cinco subcategorías permiten caracterizar técnico-pedagógicamente al buen personal docente: Adapta su metodología, Domina la evaluación, Facilita el aprendizaje, Motiva el razonamiento y Tiene objetivos definidos (ver Tabla 6).

Tabla 6: Características ténico pedagógicas

\begin{tabular}{lcc}
\hline \multicolumn{1}{c}{ Características } & Recursos & Referencias \\
\hline 4. Características técnico-pedagógicas & $\mathbf{2}$ & $\mathbf{2 9}$ \\
4.1 Adapta su metodología & $\mathbf{1}$ & \\
4.1.1 Considera la diversidad de perfiles & 1 & 1 \\
4.1.2 Considera los tiempos para aprender & 1 & 1 \\
4.1.3 Maneja estrategias diversas & 1 & 3 \\
4.1.4 Redirecciona acciones & 1 & 1 \\
\hline 4.2 Domina la evaluación & $\mathbf{2}$ & \\
4.2.1 Diseña instrumentos pertinentes & 1 & 2 \\
4.2.2 Evalúa lo que enseña & 2 & 2 \\
4.2.3 Retroalimenta los aprendizajes & 1 & 3 \\
\hline 4.3 Facilita el aprendizaje & $\mathbf{1}$ & \\
4.3.1 Genera un clima grato & 1 & 4 \\
4.3.2 Usa metodología participativa & 1 & 1 \\
\hline 4.4 Motiva el razonamiento & $\mathbf{2}$ & \\
4.4.1 Desarrolla pensamiento crítico & 2 & 4 \\
4.4.2 Relación teoría y práctica & 1 & 2 \\
4.4.3 Hace ver lo complejo fácil & 1 & $\mathbf{4}$ \\
\hline 4.5 Tiene objetivos definidos & $\mathbf{2}$ & \\
\hline
\end{tabular}

Nota: Elaboración propia. 


\section{Caracterización del buen personal docente según profesorado y grupos directivos: elementos comunes y divergentes}

En la Facultad de Educación de la Universidad Autónoma de Chile, sede Talca, tanto el profesorado como el personal directivo de carrera ejecutan labores de docencia, aunque este último ejecuta tareas de gestión y de toma de decisiones. Se reconoce en el análisis de las informaciones recogidas elementos comunes. Ambos atribuyen en prioridad características humanas al buen personal docente; no obstante, es posible observar a continuación aspectos que merecen para un grupo y para el otro una valoración diferente. Para efectos de esta comparación se consideraron solamente las subcategorías.

\section{Caracterización del buen personal docente según grupos directivos}

De acuerdo con porcentaje de referencias codificadas, para los grupos directivos la principal característica del buen personal docente es la Alteridad. La subcategoría Ejemplo de alteridad (de la categoría previa Características humanas). Es posible identificar como segunda subcategoría relevante Epistemológicamente coherente (11 referencias codificadas) compuesto por cuatro códigos: Debe producir conocimiento, Erudición en su tema, No separa teoría y práctica, Valora la teoría para comprender la práctica. La tercera subcategoría destacada según los relatos de los grupos directivos es:Tiene experticia disciplinaria (8 referencias codificadas, perteneciente a Características profesionales-institucionales); que contiene los siguientes códigos: Es visible, Se actualiza y Se especializa. A nivel pedagógico los grupos directivos solo evocan el dominio de los saberes y competencias que tienen que ver con la evaluación de los aprendizajes (Domina la evaluación: Características técnico-pedagógicas; 3 referencias codificadas).

\section{Caracterización del buen personal docente según pares}

Las categorías que reúnen el mayor número de referencias codificadas de los datos recogidos entre docentes es el Ejemplo de alteridad (Características humanas; 20 referencias codificadas). En segundo lugar, el profesorado atribuye dos características ideológicas al buen personal docente: Es capaz de trascender (12 referencias codificadas) y Es epistemológicamente coherente (9 referencias codificadas). Las subcategorías referentes a la experticia disciplinaria (Tiene experticia disciplinaria; Características profesionales-institucionales) y a la vocación (Tiene vocación; características humanas) se sitúan en tercer lugar con 7 referencias codificadas. Se destaca, igualmente, que es a partir del grupo focal de docentes desde donde surge la subcategoría Zona de tensión (Características profesionales-institucionales), compuesto por dos códigos: La evaluación docente debe adaptarse y Trabaja colaborativamente. El profesorado, en este punto, hace énfasis en la evaluación institucional y señala la necesidad de adaptarla a la complejidad del perfil ideal docente en la enseñanza superior. 
doi: http://dx.doi.org/10.15359/ree.22-2.6

URL: http://www.una.ac.cr/educare

CORREO: educare@una.cr

Es posible caracterizar al buen personal docente a partir de los principales aspectos evocados por docentes y grupos directivos (más de 15 referencias codificadas en la subcategoría). Desde esta perspectiva, el buen personal docente reúne principalmente características humanas, ideológicas y, finalmente, profesionales institucionales.

Rocha (2012) examinó la docencia universitaria desde una doble perspectiva: estudiantes y docentes. Desde la perspectiva estudiantil, la valoración de la docencia pasa por la actitud de cada docente (vocación y pasión por la enseñanza), el trato que tiene hacia el estudiantado y la enseñanza centrada en el alumnado. Mientras que los grupos docentes valoran la comunicación con los pares, el estudio permanente de su disciplina de enseñanza y el liderazgo y asertividad frente a la institución. Provisoriamente, nuestra investigación aporta nuevos atributos que tienden a aproximarse a la perspectiva del estudiantado que presenta este autor. Docentes y directivos valoran aspectos relacionales estudiantado-profesorado. La experticia disciplinaria, aunque relevante, queda postergada a un segundo plano. Cabe señalar que solo en una ocasión, los grupos docentes hicieron alusión a relación con sus pares, al indicar la dificultad que tiene para encontrarse y colaborar. Abordaremos este punto más adelante, como una Zona de tensión entre docentes y modelos institucionales.

\section{Interpretación de los resultados: Análisis de elementos relevantes}

En esta sección se busca profundizar en aquellos aspectos más relevantes para peofesorado y personal directivo cuando se trata de caracterizar la figura del buen o buena docente de universidad. Serán presentados los atributos más significativos de acuerdo con el procedimiento de análisis adoptado y detallado anteriormente. Los elementos que abarcan el mayor porcentaje de referencias son considerados como dimensiones articuladoras. No se marginalizan las subcategorías con menores referencias, sino que se asocian a los descriptores más significativos que funcionan como ejes interpretativos.

\section{El buen personal docente es un ejemplo de alteridad: Características humanas}

La noción de alteridad a la que nos remite Ricoeur (citado por Matthey y Simon, 2009) no confronta el "yo" con el "otro", sino que es este contacto con el "otro" lo que hace al sujeto evolucionar desde su complejidad humana. Es a través de ese otro ser que es posible tomar conciencia de nuestra especificidad y pluralidad. El buen equipo docente universitario es ejemplo de alteridad (45 referencias totales) tanto para docentes como para equipos directivos. La alteridad tiene relación con la capacidad del docente y de la docente de descubrir y comprender al otro ser: el estudiantado, y proyectar desde ahí su saber pedagógico. El buen equipo docente es intrínsecamente altruista (ver Tabla 2). Este se moviliza por el bienestar del estudiantado. Se compromete con este y sus necesidades de escucha y de comprensión. El personal docente 
se compromete con la formación de la persona que tiene delante de sí. (Categoría previa: Características humanas; subcategoría: Ejemplo de alteridad; código: Intrínsecamente altruista. Porcentaje de referencias $10.2 \%$ del total codificadas).

El docente tiene que tener un compromiso, responsabilidad con las personas que él está educando, que está formando. (Grupo directivo)

El buen personal docente es capaz de empatizar con sus estudiantes, de tener paciencia y de saber escuchar. Puede o persigue comprenderles. La espera y el respeto de los tiempos que el estudiantado pueden necesitar para aprender reposan sobre el principio de la empatía. Las aportaciones de Rodríguez (2007) señalan que la empatía es una habilidad social clave dentro del mundo de la docencia universitaria, pues esta participa en la interacción que se establece entre el sujeto y el mundo. Este profesional es también Próximo a sus estudiantes, pues procura construir una relación de proximidad con sus estudiantes. Establece cercanía. Asegura un buen trato y privilegia la comunicación (Categoría previa: Características humanas, subcategoría: ejemplo de alteridad; código: proximidad a sus estudiantes; $12.6 \%$ de la totalidad de las referencias codificadas).

Este modelo de profesor, donde la centralidad tendría que estar en el estudiante, el profesor convertirseen mediador, orientador, dondelo importantees la comunicación. (Grupo directivo)

Se le define también como una figura humana cercana, humilde y capaz de situarse frente al estudiantado desde sus experiencias y necesidades. "No se situé en un pedestal inalcanzable", afirma un director de carrera. La docencia se percibe, entonces, como una suerte de alianza profesorado-estudiantado en beneficio del último.

Lo ideal es que ellos sientan que nosotros estamos con ellos. (Docente)

Se considera igualmente que el buen docente Se adapta al contexto, a la realidad social, psicológica y académica del estudiantado (Categoría previa: Características humanas, subcategoría: ejemplo de alteridad; código: se adapta al contexto; $4.2 \%$ de la totalidad de las referencias codificadas).

La alteridad del personal docente, a partir de sus propias creencias, genera en el estudiantado las condiciones y le proporciona la confianza para la mejora de su autoconcepto (Héctor, 2012) y autoestima, lo cual lo conducirá a un proceso de aprendizaje exitoso. Desde la psico-sociología de R.A. Rosenthal, este fenómeno se conoce como Efecto pigmalión (Ambroise, 2003). El buen personal docente deposita en sus estudiantes altas expectativas de aprendizaje y de logro académico. En la subcategoría Produce efecto pigmaleón (Categoría previa: 
doi: http://dx.doi.org/10.15359/ree.22-2.6

URL: http://www.una.ac.cr/educare

CORREO: educare@una.cr

Características humanas, $4.8 \%$ de la totalidad de las referencias codificadas), se encuentran relatos que manifiestan esta proyección de confianza del equipo docente hacia el estudiantado:

La capacidad manifestar altas expectativas con los estudiantes. (Docentes)

Es un salto cuantitativo para muchos cuando se dan cuenta que poseen la habilidad que solamente había que despertar. (Docentes)

El buen personal docente es capaz de provocar estas mismas ambiciones en el propio estudiantado. En esta misma línea, Héctor (2012) enfatiza que un mayor compromiso del estudiantado en la solución de problemas y construcción de sus aprendizajes depende de las estrategías de motivación que activen sus docentes. Estas estrategias deben apuntar al desarrollo de la motivación intrínseca por el aprendizaje, un instrumento para la formación de mejores profesionales. Se observa, en los relatos del grupo docente entrevistado, que el buen personal docente motiva e incita las ganas de aprender y mejora la autoestima de sus estudiantes.

En síntesis, un buen o buena docente es un Ejemplo de alteridad, pues asume su función educadora desde el respeto de la existencia y experiencia del otro individuo que es el estudiantado. Es un buen o buena docente quien responde desde su propia humanidad (empatía y humildad) a las necesidades educativas del estudiantado. Es capaz de depositar confianza en este y conseguir que el mismo alumnado crea en sus potencialidades y posibilidades para aprender lo que el currículo explícito e implícito le presenta como desafío.

\section{El buen o buena docente es epistemológicamente coherente: Características ideológicas}

El buen o buena docente es epistemológicamente coherente (Categoría previa: Características ideológicas). El personal docente debe mostrar experticia en su disciplina y debe ser capaz de producir conocimiento nuevo, en lo disciplinario y en lo pedagógico. Para esto, tiene la capacidad de hacer dialogar la teoría y la práctica, haciendo visible para sus estudiantes esta interdependencia de las dos formas de conocimiento. Se requiere, entonces, una comprensión acabada de la complejidad del contenido disciplinario que es objeto de enseñanza.

En realidad, en la docencia, yo creo que la relación entre teoría y práctica es fundamental, y una se nutre con la otra, ahora yo soy una convencida que, mientras más teoría conozco más bien puedo interpretar mi practica y más bien puedo conocer los problemas que se viven en la práctica, yo soy una convencida de eso. (Grupos directivos) 
Manifiesta una relación abierta con el saber (Categoría previa: Características ideológicas; subcategoría: Mantiene una relación abierta con el saber, $4.8 \%$ de la totalidad de las referencias codificadas), se encuentra en permanente búsqueda de nuevos conocimientos, no desde la obligación institucional, sino desde su propia curiosidad por saber. De manera autónoma investiga, analiza y construye conocimiento. Es así como el buen personal docente es definido como sujeto reflexivo, para quien los saberes dialogan entre sí. Su mirada acerca del conocimiento es compleja y no segmentada.

Tiene que ser una persona curiosa para siempre estar buscando cosas para los estudiantes. (Docentes)

Freire (2006), en Pedagogía de la autonomía. Saberes necesarios para la práctica educativa, considera como saberes fundamentales de la práctica docente el pensamiento crítico. No es solo la curiosidad -curiosidad ingenua- la que permite a los sujetos aproximarse al objeto cognoscible de forma crítica, sino su curiosidad epistemológica: inquietud indagadora, metódicamente rigurosa, que es la fuente vital de la creatividad, social e históricamente construida y reconstruida. La práctica docente crítica, entonces, "encierra el movimiento dinámico, dialéctico, entre el hacer y el pensar en el hacer" (Freire, 2006, p. 39). Desde esta perspectiva, la enseñanza universitaria exige al profesorado transformarse para transformar o superar sus formas de conocer y de intervenir en el mundo, para superar esas mismas formas de aprender y de aprehender el mundo en sus estudiantes.

Así, el buen o buena docente es capaz de trascender (Categoría previa: Características ideológicas; subcategoría: Es capaz de trascender, $10.8 \%$ de la totalidad de las referencias codificadas), es decir, es capaz de generar cambios en ámbitos académicos y personales. Las actitudes, los valores que el equipo docente porta son fuente de inspiración para el estudiantado. El buen docente debe ser un modelo profesional, humano y social.

Usted es capaz de cambiar la vida del alumno. Este cambio es académico, es personal, es social, es un montón de cosas. No solo es una nota, hay personas que no tienen una buena nota, pero están súper feliz, súper satisfechos con lo que están recibiendo, y están satisfechos con el resultado final y esto tiene que ver con el cambio. (Docentes)

La última subcategoría de las características ideológicas del buen personal docente describe una suerte de principio fundador de la docencia: tiene vocación (Categoría previa: Características ideológicas; subcategoría: Tiene vocación, $4.2 \%$ de la totalidad de las referencias codificadas). El buen o buena docente profesa amor hacia su trabajo de enseñanza, es también, de esta manera, como puede despertar ese sentimiento en el estudiantado. 
doi: http://dx.doi.org/10.15359/ree.22-2.6

URL: http://www.una.ac.cr/educare

CORREO: educare@una.cr

Amor por lo que uno hace por la disciplina. A lo mejor es un poco poético, pero si uno no tiene eso [amor], vas a cumplir medianamente o de buena manera, no sé... Pero sin ganas, sin ese amor por lo que uno hace, no va a haber superación y no se le va a poder transmitir a los estudiantes lo que uno en realidad quiere transmitir, que en realidad va más allá que el conocimiento que está en los libros". (Docente)

Cabe señalar que este aspecto solo emerge en el grupo focal de docentes y no el realizado con personal directivo. Emocional, social e intelectualmente con gran compromiso con su labor de enseñanza, el buen o buena docente trasciende y es susceptible de hacerlo si tiene, para sí, la propia capacidad de trascender en sus formas de conocer.

\section{El buen o buena docente hace carrera y tiene un buen curriculum profesional: Características profesionales e institucionales}

La experticia disciplinaria aparece, igual que en otros estudios, como un componente importante, pero no prioritario, del perfil docente universitario de calidad. La formación continua en el ámbito de su área de conocimientos se asocia con la capacidad docente de actualizar permanentemente sus saberes disciplinarios. (Categoría previa: Características profesionalesinstitucionales; subcategoría: Tiene experticia disciplinaria; $9 \%$ de la totalidad de las referencias codificadas).

El buen personal docente no es generalista, sino especialista que domina progresivamente a través de estas trayectorias de formación un conocimiento acabado de su disciplina. Esta experticia le hace objeto de reconocimiento social.

Está en contacto con diferentes redes, [es] una persona visible en sus competencias y no enajenado, a quien nadie conoce. Tiene que ser una persona visible en el ámbito de su competencia, en los diferentes medios. (Directivos)

La segunda subcategoría Tiene trayectoria profesional integral (Categoría previa: Características profesionales-institucionales; subcategoría: Tiene trayectoria profesional integral, $4.2 \%$ de la totalidad de las referencias codificadas) define al buen personal docente como profesional cuya experticia se encuentre acreditada, por un lado, por diplomas, y, por otro, por su experiencia en el campo de su saber.

Los mejores profesionales que se han contratado son porque tienen las tres cosas ... o son expertos en su área, han hecho una buena práctica y tienen experiencia a lo largo de los años. (Grupos directivos) 
El grado académico se reconoce en las intervenciones de los grupos directivos como un indicador de calidad que rara vez engaña en cuanto a la calidad del desempeño docente.

la universidad establece que debe ser un profesional competente, cinco años de experiencia mínimo, con pos-grado o pos-título. Cabe la casualidad a lo largo de mi experiencia directiva, que entre mayor conocimiento, títulos y grados y perfeccionamientos que se tiene, se es mejor profesor. Es decir, no es casualidad que una persona que tiene más competencias o más experiencia es más competente profesionalmente". (Grupos directivos)

Sin embargo, los diplomas son asociados a la experiencia profesional, en el caso de docentes de la formación inicial de profesorado de enseñanza general y disciplinaria, el buen o buena docente es quien ha ejercido como tal también.

Tener experiencia en el área que uno enseña. Yo en mi caso soy profesor básico, para mí fue trascendental haber tenido la experiencia que tuve en aula con niños, para poder traspasaresa experiencia a los estudiantes y también hacerles sentir que los contenidos que están tratando en las diferentes temáticas que se den, les sirven para tal y cual momento. (Docentes)

Ser buen o buena docente no constituye solo en una acumulación de diplomas o años de ejercicio profesional en el aula escolar, es reunir estos dos aspectos.

El análisis de los grupos focales, docentes y directivos, manifiesta dos tensiones que se producen entre el ejercicio de la docencia y las exigencias institucionales. En el análisis este fenómeno fue categorizado como Zona de tensión (Categoría previa: Características profesionales-institucionales; subcategoría: Zonas de tensión, $5.4 \%$ de la totalidad de las referencias codificadas). Reconocen los grupos entrevistados que la fragmentación de los contenidos curriculares y las formas de trabajo individualizado limitan la calidad del desempeño docente; es decir, docentes capaces de transitar de manera comprensiva por los distintos ámbitos del currículo y de establecer conexiones y coherencias pedagógicas en su labor docente. Por esto, el buen personal docente está llamado a trabajar colaborativamente (Categoría previa: Características profesionales-institucionales; subcategoría: Zonas de tensión; código: Trabaja colaborativamente; $0.6 \%$ de la totalidad de las referencias codificadas).

Existe mucho divorcio respecto a los profesores que hacen clase acá, en el sentido de que no hay dialogo, no hay conversación, y si no hay dialogo, y no hay conversación ... si yo estoy en una carrera que es de enseñanza media y que hay especialidades, el profesor de una especialidad Historia Universal hace Historia Universal. El que hace Geografía Humana hace Geografía Humana. Por lo tanto, no hay dialogo, no hay conversación, por lo tanto, ese profesor no se impregna de lo que está pasando en el currículo, no se impregna de lo que está pasando en 
doi: http://dx.doi.org/10.15359/ree.22-2.6

URL: http://www.una.ac.cr/educare

CORREO: educare@una.cr

el taller. Se impregna también poco y casi nada de lo que hace el otro. Al final cada profesor termina en su parcela, por tanto, no hay integralidad y si en los profesores no hay integralidad tampoco vamos a lograr integralidad en el estudiante, esa es mi percepción. (Grupos directivos)

Por otro lado, los grupos docentes estiman que las evaluaciones institucionales de calidad de la docencia por parte de estudiantes, personal directivo y autoevaluación se limitan a evaluar aspectos administrativos y dejan fuera otras dimensiones del ejercicio profesional. La evaluación docente debe adaptarse (Categoría previa: Características profesionales-institucionales; subcategoría: Zonas de tensión; código: La evaluación docente debe adaptarse; $4.8 \%$ de la totalidad de las referencias codificadas) al perfil complejo del buen personal docente. La expresión de estas tensiones, si bien son menos relevantes, en cuanto a la ocurrencia temática y porcentaje de referencias, ellas abordan aspectos que merecen la pena ser explorados. Indagar sobre las condiciones y contextos que favorecen y valorizan la buena docencia, interrogar las formas de gestion universitaria y curricular son algunas pistas que permitirían prolongar la investigación en torno a la figura del buen personal docente.

\section{El buen o buena docente centra su pedagogía en el estudiantado: Características técnico-pedagógicas}

El buen personal docente posee cuatro características técnico-pedagógicas: Adapta su metodología, domina la evaluación, facilita el aprendizaje, motiva el razonamiento, tiene objetivos definidos. Por una parte, se describe como profesional capaz de entender la diversidad humana, esencialmente en el seno de su grupo de estudiantes. Adapta su metodología de enseñanza (Categoría previa: Características técnico-pedagógicas; subcategoría: Adapta su metodología; $3 \%$ de la totalidad de las referencias codificadas), pues considera esta diversidad como un elemento constitutivo de su trabajo pedagógico.

La Facultad de Educación de la Universidad Autónoma de Chile tiene la responsabilidad de formar de manera integral al futuro profesorado, acogiendo distintos tipos de estudiantes, con diferentes intereses, habilidades y talentos. Esto se hace patente en la elección que estos grupos hacen de sus carreras de formación pedagógica (Educación Parvularia, de Educación General Básica y docentes de asignatura: Lenguaje y Comunicación, Lengua Inglesa, Historia y Geografía, Artes Visuales, Matemáticas, Educación Física y Salud). Los docentes y las docentes reconocen que estudiantes de ciertas carreras tendrán menos dificultad para responder a las exigencias académicas transversales que tienen que ver con el manejo de la lengua. Como lo señala Zabalza (2013), la expresión oral y escrita son competencias básicas de la formación universitaria. Los cuerpos docentes asumen que estudiantes de carreras pedagógicas de corte humanista y sociales cuentan con competencias en este sentido, o al menos las desarrollan con más facilidad que estudiantes de otras carreras pedagógicas. 
Un alumno de lenguaje va a tener una competencia mayor que un alumno de artes visuales, por lo tanto, del punto de vista pedagógico uno tiene que adecuar de una u otra manera la estrategia metodológica que va a ocupar a los alumnos de distintos perfiles. (Docentes)

La capacidad docente de adaptar su metodología de enseñanza tiene relación con la necesidad introducir y acompañar al estudiantado en la cultura universitaria, en la que el lenguaje es una herramienta fundamental y en donde se aspira al desarrollo de aprendizajes de nivel superior. Coulon (1990), en sus investigaciones en torno al oficio de estudiantado universitario, indica que la permanencia y éxito académico depende del nivel de aculturación del estudiantado a la vida universitaria. Atender a los ritmos implica un mejor conocimiento por parte del equipo docente de sus estudiantes y de la manera en que construyen su aprendizaje para movilizarles desde ahí hacia los objetivos de la enseñanza en contexto universitario.

Conociendo a mis alumnos, se cómo aprenden mis alumnos. [Si sé] cómo aprenden mis alumnos voy a ser capaz de poder desarrollar mis actividades de aprendizaje, direccionar mis actividades de aprendizaje dentro de la sala de clase hacia el desarrollo de habilidades o dominios cognitivos de nivel superior. (Docente)

El buen personal docente Domina la evaluación (Categoría previa: Características técnicopedagógicas; subcategoría: Domina la evaluación; $4.2 \%$ de la totalidad de las referencias codificadas). Tiene las competencias para diseñar y proponer al estudiantado instrumentos y procedimientos de evaluación pertinentes.

Tener la experticia en cuanto al diseño y a la aplicación del instrumento de evaluación. (Docentes)

De la misma manera, el dominio de la evaluación consiste en la coherencia entre lo que se enseña y la forma en la que se evalúa. Es capaz de situar la evaluación en la prolongación de los aprendizajes y no como una ruptura.

Un buen profesor es aquel que es un profesor consecuente, que evalúa lo que enseña y que además es capaz de hacer una retroalimentación, donde independientemente del resultado que ellos tengan en las evaluaciones, que en esa evaluación se refleje efectivamente lo que el profesor enseñó. (Grupo directivo)

La subcategoría Facilita el aprendizaje emerge del discurso de docentes (Categoría previa: Características técnico-pedagógicas; subcategoría: Facilita el aprendizaje; $3 \%$ de la totalidad de las referencias codificadas). Para el grupo docente, el buen maestro o la buena maestra es capaz 
doi: http://dx.doi.org/10.15359/ree.22-2.6

URL: http://www.una.ac.cr/educare

CORREO: educare@una.cr

de generar climas que faciliten el aprendizaje. Estos espacios de aprendizaje están orientados hacia una práctica pedagógica de tipo activo-participativo que genere, en el estudiantado, una experiencia grata de aprendizaje.

Metodología tipo participativa, o pedagogía colaborativa que son elementos básicos para realizar una docencia de calidad. (Docentes)

Conducir al estudiantado hacia el desarrollo de un pensamiento crítico, a la comprensión de las relaciones que se establecen entre las distintas formas del saber (lo teórico, lo práctico) constituye la categoría Motiva el razonamiento (Categoría previa: Características técnicopedagógicas; subcategoría: Motiva el razonamiento; $4.2 \%$ de la totalidad de las referencias codificadas). El buen personal docente asume un despertar intelectual en el estudiantado por sobre la transmisión de conocimientos.

Tú puedes lograr un sujeto critico reflexivo, en la medida que tu generes los ambientes, y lo hagas pensar. (Grupos directivos)

Martínez (2011) dirá que es propio de estos tiempos que sea el alumnado el que, a través de la información existente, partirá en la búsqueda de soluciones. “El mismo Einstein solía decir que 'el arte más importante de un maestro es saber despertar en sus alumnos la alegría de conocer y crear"' (p. 121).

Finalmente, el buen o buena docente tiene objetivos definidos (Categoría previa: Características técnico-pedagógicas; subcategoría: Tiene objetivos definidos; $2.4 \%$ de la totalidad de las referencias codificadas). Esto indica que el personal docente ideal sabe hacia dónde quiere llevar a sus estudiantes.

Visión general de donde quiero llegar, por ejemplo, la perspectiva que uno tiene de ese grupo que está aquí, como yo tengo que ver para terminarlo, yo en la asignatura que yo tengo cual es la perspectiva o la propuesta a esa asignatura, entonces la visión final de como seria de cómo debería ser y para nosotros se perdería la gracia, pero la perspectiva final. (Docentes)

Elbuen o buena docente se proyecta en el proceso de aprendizajejunto con el estudiantado.

\section{Conclusiones}

En Chile, a partir de los estudios de Ávalos $(2002,2014)$ y Ávalos y Nordnflycht $(1999)$ y Ávalos, Cavada, Pardo y Sotomayor (2010) se ha instalado la idea de que el profesorado es una figura central en el proceso de enseñanza-aprendizaje, cuestión que llevó a que, a mediados 
de la década del 90, el gobierno de la época anunciara que enfrentaría el desafió de revitalizar la profesión docente, de tal manera que el Ministerio de Educación anunció el programa Fortalecimiento de la Formación Inicial Docente (FFID), que comenzó a operar el año 1997 por un periodo de cinco años. Lo anterior, en el bien entendido de que las nuevas generaciones de maestros y maestras deben responder a la creciente complejidad del escenario mundial $y$, particularmente, a los retos desplegados por la reforma en marcha como la nueva matriz curricular y la nueva concepción referida a prácticas pedagógicas.

Luego de dos décadas de implementación de políticas en favor de la docencia, se concluye que han dado pasos significativos, no obstante, la labor del profesorado continúa siendo el factor decidor en la relación pedagógica, en el aprendizaje estudiantil y, en definitiva, en la misión de desarrollo humano que tiene el sistema educativo en los diferentes niveles de enseñanza.

Finalmente, podemos afirmar que el tema del profesorado continuará en la discusión pública, debido a su papel decidor en el proceso de mejora en la calidad de los aprendizajes de los niños, niñas y adolescentes, cuestión que representa la gran preocupación de la reforma educacional en sus etapa presente, junto al desafío, no menor, de lograr crecientes niveles de equidad en el sistema.

El objetivo de esta investigación era caracterizar al buen o buena docente universitario desde la perspectiva del profesorado y personal directivo de carrera de la formación de profesores. A través de datos recogidos mediante la técnica de grupos focales, se buscó poner en discusión la valoración del personal docente universitario con el propósito de describir esta figura tomando a los propios actores como referentes. Entonces, es posible atribuir al buen o buena docente de la universidad cuatro características. Sus características humanas exaltan las dimensiones valóricas y actitudinales del profesorado universitario, pues estas le permiten construir relaciones interpersonales humanamente simétricas con sus estudiantes. Las características ideológicas hacen referencia al actuar profesional y las normas, teorías y creencias que regulan su acción pedagógica. Las características profesionales e institucionales abordan su trayectoria profesional y académica, que lo legitima como sujeto formador. Por último, las características técnico-pedagógicas subrayan los saberes, procesos, condiciones y artefactos con los que docentes contribuyen al acto de formación del estudiantado. Docentes y personal directivo valoran aspectos relacionales del estudiantado-profesorado. La experticia disciplinaria, aunque relevante, queda postergada a un segundo plano.

Un primer estudio realizado con estudiantes arroja como principales rasgos característicos del buen o buena docente: el dominio del contenido y la calidad de la metodología de la enseñanza, en cuanto al perfil pedagógico (Merellano-Navarro et al., 2016). Esta parte de la investigación centrada en decentes y equipos directivo de la misma facultad, Facultad de Educación de la Universidad Autónoma de Chile, en su sede Talca, pone en relieve otros 
doi: http://dx.doi.org/10.15359/ree.22-2.6

URL: http://www.una.ac.cr/educare

CORREO: educare@una.cr

aspectos: lo humano y lo ideológico, por sobre lo técnico-pedagógico y lo institucional. En la era de los TIC, de la globalización y del alto flujo de la información, el cuerpo docente reinstituye la educación como un fenómeno pedagógico en un sentido más amplio y trascendental. Chalmel (2011) hablará de la fe del pedagogo para expresar un credo compartido por poblaciones laicas, marxistas, libertarias en todo proyecto educativo. El buen o buena docente, tanto para estudiantes como para docentes y personal directivo, es un sujeto ético. Desde esta característica primigenia, el buen o buena docente construye primero, en sí, su pensar crítico, su constructo pedagógico y profesional epistémico y disciplinario. No trasciende de otra forma y no consigue hacerlo tampoco fuera de una relación ante todo humana.

\section{Límites y perspectivas}

Esta investigación intentó aproximarse a la concepción de los propios grupos docentes y personal directivo sobre lo que es un buen o buena docente de universidad. El trabajo realizado esboza pistas de reflexión que valdría la pena triangular con otros métodos. En este sentido, es importante destacar que la primera parte de esta investigación que recogió la percepción del estudiantado, lo hizo a partir de dos instrumentos: primero un cuestionario y luego una entrevista. Los cuestionarios contaban con ítems referidos al ámbito pedagógico. Los grupos focalizados abordaron los aspectos más significativos arrojados por el cuestionario. Valdría la pena interrogarse si los cuerpos docentes, quienes participaron en grupos focales de discusión abierta, caracterizarían al buen o buena docente de la misma forma, con un instrumento estructurado en ítems predeterminados. También resulta necesario explorar este fenómeno con una muestra más amplia en áreas de formación en educación, así como también en otras formaciones profesionales. Por último, queda igualmente la tarea de indagar sobre la relación entre estas concepciones sobre la enseñanza en el equipo docente, la práctica pedagógica y los niveles de desempeño del estudiantado.

\section{Referencias}

Ambroise, G. (2003). Aux sources de l'effet Pygmaleon. Éduquer, 5. Recuperado de http://journals. openedition.org/rechercheseducations/218\#quotation

Ávalos, B. (2002). Profesores para Chile, historia de un proyecto. Santiago: Ministerio de Educación. Recuperado de http://docplayer.es/14501497-Beatrice-avalos-profesores-para-chilehistoria-de-un-proyecto.html

Ávalos, B. (2014). La formación inicial docente en Chile: Tensiones entre políticas de apoyo y control. Estudios Pedagógicos, 40(Especial 1), 11-28. Recuperado de https://scielo.conicyt. cl/pdf/estped/v40nEspecial/art02.pdf 
Ávalos, B.y Nordenflycht, M.E.(Eds.). (1999). Laformación de profesores: Perspectivasyexperiencias. Santiago: Santillana.

Ávalos, B., Cavada, P., Pardo, M. y Sotomayor, C. (2010). La profesión docente:Temas y discusiones en la literatura internacional. Estudios Pedagógicos, 36(1) 235 -263. Recuperado de http:// www.redalyc.org/articulo.oa?id=173516404013

Blais, M. y Martineau, S. (2006). L'analyse inductive générale: Description d'une démarche visant à donner un sens à des données brutes. Recherches Qualitatives, 26(2), 1-18. Recuperado de http://www.recherche-qualitative.qc.ca/documents/files/revue/edition reguliere/ numero26(2)/blais et martineau final2.pdf

Casero, A. (2010). ¿Cómo es el buen profesor universitario según el alumnado? Revista Española de Pedagogía, 68(246), 223-242. Recuperado de http://www.jstor.org/stable/23766298

Gibezzi, V. (2010). Aportes desde la práctica educativa constructivista. Reflexión académica en diseño \& comunicación, 13(11), 67-68. Recuperado de http://fido.palermo.edu/servicios dyc/publicacionesdc/archivos/127 libro.pdf

Chalmel, L. (2011). La pédagogie au risque de la foi. En M. Soëtard y G. Le Bouëdec, (Dir.), La foi du pédagogue (pp. 1-20). Paris: Don Bosco. Recuperado de http://www.ere-oca.com/ documents/document 473.pdf

González, E., Herrera, R. H., Zurita, R. (2008). Formación basada en competencias: Desafíos y oportunidades. En Cinda (Ed.), Diseño curricular basado en competencias y aseguramiento de la calidad en la educación superior (pp. 16-28). Santiago: Cinda. Recuperado de http:// www.upch.edu.pe/rector/dugec/images/files/biblioteca/39.PDF

Coulon, A. (1990). Le Métier d'étudiant: Approches éthnométhodologique et institutionnelle de l'entrée dans la vie universitaire. Lille, France: Atelier National de Reproduction des Thèses.

De-Juanas, A., Ezquerra, Á. y Martín, R. (2016). Tendencias metodológicas en los docentes universitarios que forman al profesorado de primaria y secundaria. Revista Brasileira de Educação, 21(65), 391-409. doi: https://doi.org/10.1590/S1413-24782016216521

Delors (Preside). (1997). Informe a la Unesco de la Comisión internacional sobre la educación para el siglo veintiuno. La Educación encierra un tesoro. Madrid: Santillana Ediciones Unesco. Recuperado de http://www.unesco.org/education/pdf/DELORS S.PDF

Demougeot-Lebel, J. (2014). Enseignants-chercheurs de la génération Y: Incidence sur les pratiques pédagogiques? Revue Internationale de Pédagogie de L'enseignement Supérieur, 30(3). Recuperado de http://ripes.revues.org/883 
doi: http://dx.doi.org/10.15359/ree.22-2.6

URL: http://www.una.ac.cr/educare

CORREO: educare@una.cr

Fernández, M. A.y González, S. (2012). El perfil del buen docente universitario: Una aproximación en función del sexo del alumnado. REDU: Revista de Docencia Universitaria, 10(2), 237-249. doi: https://doi.org/10.4995/redu.2012.6106

Francis, S. (2006). Hacia una caracterización del docente universitario «excelente»: Una revisión a los aportes de la investigación sobre el desempeño del docente universitario. Educación, 30(1), 31-49. Rdoi: https://doi.org/10.15517/revedu.v30i1.1793

Freire, P. (2006). Pedagogía de la autonomía. Saberes necesarios para la práctica educativa (11a ed., trad. G. Palacios). México: Siglo XXI.

García, W. y Martín, M. A. (2013). Hermenéutica y pedagogía: La práctica educativa en el discurso sobre la educación. Pulso, 36, 55-78. http://dspace.uah.es/dspace/handle/10017/19801

Hamui-Sutton, A. y Varela-Ruiz, M. (2013). La técnica de grupos focales. Investigación en educación médica, 2(5), 55-60. doi: https://doi.org/10.1016/\$2007-5057(13)72683-8

Héctor, E. F. (2012). La motivación como sustento indispensable del aprendizajeen los estudiantes universitarios. Pedagogía Universitaria, 17(4), 13-27. Recuperado de http://cvi.mes.edu.cu/ peduniv/index.php/peduniv/article/view/39

Luna-Serrano, E., Vallle-Espinosa, M. y Osuna-Lever, C. (2010). Los rasgos de un "buen profesional", según la opinión de estudiantes universitarios en México. Revista electrónica de investigación educativa, Número especial, 1-14. Recuperado de http://www.redalyc. org/articulo.oa?id=15518482006

Martínez, M. (2011). La docencia universitaria hoy: Metas, técnicas y desafíos. Argos, 28 (55), 108124. Recuperado de http://bdigital.ula.ve/storage/pdf/argos/n55/art05.pdf

Martínez, M., García, B. y Quintanal, J. (2006). El perfil del profesor universitario de calidad desde la perspectiva del alumnado. Educación, 21(9), 183-198. doi https://doi.org/10.5944/ educxx1 9.0.325

Matthey, M. y Simon, D.-L. (2009). Altérité et formation des enseignants: Nouvelles perspectives. Lidil, 39, 5-18. Recuperado de http://lidil.revues.org/2733

Merellano-Navarro, E., Almonacid-Fierro, A., Moreno-Dona A. y Castro-Jaque, C. (2016). Buenos docentes universitarios: ¿Qué dicen los estudiantes? Educação E Pesquisa, 42(4), 937-952. doi: https://doi.org/10.1590/s1517-9702201612152689

Palma, C. (2013). La privatización de la educación superior en Chile: Procesos de masificación y reproducción social. Sociedad Hoy, 24, 119-140. Recuperado de http://www.redalyc.org/ articulo.oa?id=90231580010 
Rocha, R. (2012). La docencia universitaria desde la perspectiva de los alumnos frente a la de los profesores. Revista Innovación Educativa, 12(58), 91-118. Recuperado de http://www. redalyc.org/articulo.oa?id $=179424061006$

Rodríguez, L. (2007). Habilidades sociales y docencia universitaria. En Fonseca y J I. Aguaded (Dirs.), Enseñar en la universidad. Experiencias y propuestas de docencia universitaria (pp. 3750). España: Netbiblo.

Saldaña, J. (2009). The coding manual for qulitative researchers. Los Ángeles: SAGE.

Simons, H. (2011). El estudio de caso: Teoría y práctica. Madrid: Ediciones Morata.

Tardif, M. (2004). Los saberes del docente y su desarrollo profesional. Madrid: Narcea Ediciones.

Ventura, M. C., e Neves, M. M., Loureiro, C. R., Frederico-Ferreira, M. M. y Cardoso, E. M. P. (2011). O "bom professor" - Opinião dos estudantes. Revista de Enfermagem Referência, 5, 95-102. doi: https://doi.org/10.12707/RIII1167

Voynnet, C., (2012). Ce que "analyse de données qualitatives" veut dire. Revue Internationale de Psychologie, 18(44), 71-88. Recuperado de https://www.cairn.info/revue-internationalede-psychosociologie-2012-44-page-71.htm

Zabalza, M. (2013). Escribir en la universidad. Educação, 38(1), 15-39. Recuperado de http:// www.redalyc.org/articulo.oa?id=117125620002 